\title{
Causas de la crisis de la profesión médica en Chile
}

\author{
Antonio Vukusich C, Francisco Olivari P, \\ Nelly Alvarado A, Gloria Burgos M.

\section{Causes of the crisis of medical profession in Chile}

There is a growing dissatisfaction among physicians towards their professional practice. This article tries to identify causes of this dissatisfaction and to explain the fears that physicians experience during their practice. The authors pose the hypothesis that physicians have non resolved internal problems with the application of technological advances and that medical practice has not changed along with the dramatic social changes that have occurred in the last decades. Medical organizations work in a confusing environment. Considering the main characteristics of medical work, the causes of difficulties and the reasons to explain the slowness of physicians to identify the problems and react, are analyzed. Finally some solutions are proposed to overcome this crisis (Rev Méd Chile 2004; 132: 1425-30).

(Key Words: Clinical practice patterns; Defensive medicine; Ethics, medical; Knowledge, attitudes, practice)

Recibido el 11 de agosto, 2004. Aceptado el 16 de agosto, 2004.

Dirección Médica. Clínica Dávila.

L a palabra crisis se aplica a situaciones complejas en las que no se vislumbra una salida. Por esto, pensamos que es adecuada para caracterizar las condiciones en las que el médico clínico realiza hoy su trabajo: un escenario de expectativas crecientes, muy difíciles de satisfacer, aun sin considerar los problemas estructurales y de arrastre del sistema de salud. En esta crisis, la imagen del médico ha sufrido un deterioro y la práctica clínica -habitualmente llena de satisfaccionessuele transcurrir en medio de la insatisfacción, la ansiedad e incluso el temor. Numerosos artículos analizan las causas del descontento de los médicos en el mundo desarrollado ${ }^{1-4}$. En nuestro país se han publicado tanto descripciones como intentos por comprender el problema ${ }^{5-7}$.

Correspondencia a: Dr. Antonio Vukusich C. Recoleta 464. Recoleta, Santiago. Fax: 7308885. E mail: dirmedica@davila.cl
La situación tiene aspectos paradójicos. El «material» humano que se forma en las Escuelas de Medicina es de reconocida calidad. Los médicos -mayoritariamente- son profesionales competentes, altamente entrenados, autocríticos y de gran capacidad de trabajo. Chile mantiene indicadores tradicionales de salud competitivos con países cuyos presupuestos per cápita para el sector son 10 a 20 veces mayores que el nuestro. Sin embargo, la sociedad nos critica abiertamente y los profesionales ajenos al área se sienten en condiciones de proponer soluciones para nuestro sector. En resumen: por primera vez en los últimos 50 años los médicos enfrentamos serios problemas.

$\mathrm{Si}$ los indicadores tradicionales de salud parecen superados por el nuevo perfil epidemiológico de la población, podemos concordar en que el sistema de provisión de servicios de salud es anacrónico y que debería actualizarse. Pero, más 
allá de los problemas de nuestro sistema de salud, lo que realmente parece estar en crisis es la forma en que se practica la medicina misma y esto debe preocuparnos, ya que tenemos una gran responsabilidad en su solución.

Nuestro entorno laboral ha cambiado profundamente, al haber sido superado el Estado como principal empleador. Las posibilidades laborales - que se limitaban al médico funcionario- se han ampliado, estableciéndose una gran variedad de relaciones laborales. Sin embargo, y sin relación alguna con el tipo de contrato que afecte a los médicos, la naturaleza misma de su práctica, es decir, la manera de resolver los problemas de las personas enfermas, ha permanecido intacta, oculta y protegida por capas de lenguaje que no dejan ver lo esencial. Se ha señalado que el pragmatismo, la masificación, las mediciones de la productividad, la sobrevaloración de la relación costo-beneficio y de la eficiencia aparecen como elementos inherentes a la práctica médica actual ${ }^{8}$. Cabe preguntarse: jpor qué nuestro trabajo tiene hoy estas características?

\section{LA IMPORTANCIA DEL CONTEXTO}

Las características de la práctica médica han variado a lo largo de la historia, según cómo fue interpretado el hecho de enfermar. La enfermedad ha sido vista como un castigo (por faltas personales o sociales); como una sprueba» (que se puede pasar o no); o como una manifestación de la voluntad o la presencia de Dios. Por lo tanto, la actitud de cualquier sociedad con sus enfermos depende siempre de su visión del hombre sano, es decir, de una respuesta a la pregunta: ¿qué significa ser hombre? Toda antropología supone, a su vez, una cosmología, una determinada visión del mundo como totalidad.

En nuestro tiempo, vivimos una transición entre dos épocas: la modernidad y lo que suele llamarse post-modemidad ${ }^{9}$. Están bajo escrutinio nada menos que nuestra visión del mundo y nuestra visión del hombre. Pero a diferencia de otras épocas de crisis, generalmente breves, experimentamos la actual como un continuo, como una forma de vivir. La transición se precipitó en el siglo XX debido a: 1. La constatación de un aspecto irracional y destructivo en el hombre; 2. La declinación de la influencia de las religiones y las ideologías, sin las cuales, en un escenario valónico diverso, sólo el éxito tangible e inmediato es aceptado como critenio para distinguir lo verdadero de lo falso; y 3. El fin de la utopía iniciada en el siglo XVII que creía posible un conocimiento completo y acabado a partir de las ciencias ${ }^{10-13}$.

Al conocerse las limitaciones de los sistemas axiomáticos y la incertidumbre en el conocimiento del mundo material, las ciencias han pasado a tener sólo un rol instrumental en el manejo del mun$\mathrm{do}^{14,15}$. No existe un modelo único, ni bien definido, para comprender qué es el hombre $\mathrm{y}$, dada la ausencia de certezas, el deseo y la confianza en el poder de la voluntad humana parecen dominar en el ampliamente extendido agnosticismo occidenta ${ }^{16}$. Con él conviven el hedonismo, la inmediatez y una esperanza sin límites en las posibilidades de la medicina. No se acepta la enfermedad ni la necesidad de la muerte, que es vista como un fracaso (de alguien o de algo) y se quiere utilizar al médico para propósitos que van más allá del cuidado de los enfermos. En este ambiente, eso que llamamos salud -y cuya definición expresa otra utopía- ha dejado de ser un don para convertirse en un derecho ampliamente promocionado. Al identificarse la salud con un derecho se establece, tácitamente, un nuevo «contrato» entre médico y paciente que, como veremos, resulta imposible de cumplir por parte del médico. En la relación médico-paciente que conocimos, el médico podía cumplir estudiando intensamente para dar a sus pacientes el mejor cuidado de que era capaz. A cambio obtenía un trabajo seguro, autónomo y razonablemente remunerado en un ambiente de deferencia y comprensión. El nuevo escenario tiene profundas consecuencias. No sólo el énfasis en la satisfacción de las expectativas y el punto de vista del paciente. También la evaluación de aspectos no técnicos del trabajo médico.

\section{FACTORES DETERMINANTES DEL TRABAJO MÉDICO}

Lo propio ( interno») del trabajo médico ha sido el cuidado de las personas enfermas. Sus características varían dependiendo de factores «externos»al quehacer médico mismo. Un factor fundamental es el nivel científico-tecnológico. Los otros determinantes son: la visión de los problemas morales; el marco jurídico en que se desarrolla la praxis; el modelo y los mecanismos de regulación económica; los propósitos de las organizaciones médicas 
y, finalmente, los objetivos político-sanitarios del Ministerio de Salud. Si aceptamos que los factores determinantes son los señalados, la siguiente hipótesis es plausible:

El trabajo médico es a menudo tan absorbente que nos ha impedido entender e incorporar los dramáticos cambios ocurridos con los factores que lo determinan $\mathrm{y}$, por lo tanto, se realiza en conflicto con todos ellos, en un ambiente de confusión de las organizaciones médicas.

El análisis que sigue se dividirá en cuatro partes: 1. Caracterización de la práctica médica; 2 . Los problemas con los factores determinantes; 3. Cómo llegamos a la situación actual y; 4. Condiciones para avanzar en la solución de los problemas.

\section{CARACTERIZACIÓN DE LA PRÁCTICA MÉDICA}

La insuficiente racionalidad de la práctica médica Nuestro trabajo tiende a ser conservador y, a pesar del desamollo tecnológico, hay una enorme dificultad para incorporar cambios de fondo en la enseñanza y la práctica clínica, porque tenemos poco tiempo para reflexionar sobre lo que hacemos y cómo lo hacemos ${ }^{17}$. Aún tomamos decisiones usando evidencia anacrónica, opiniones de autoridades o experiencias personales no publicadas ni sometidas a crítica y recurrimos poco a mediciones de los resultados finales de los procesos de atención.

El sentido de independencia profesional produce gran variedad de soluciones para un mismo problema que no pueden justificarse por diferencias en los resultados ni por la variabilidad biológi$\mathrm{ca}^{18}$. Por otra parte, tenemos dificultades para cumplir las normas y protocolos, lo que obliga a la centralización del control de la atención. Los medios de diagnóstico no se reemplazan con el avance tecnológico sino que se suman. Se hace lo que se hacía antes más lo que se debería hacer ahora porque no sabemos -a partir del análisis de nuestros propios datos- cuáles prácticas son más adecuadas y las experiencias de otros lugares tienden a modificarse sin aplicar un criterio común.

Carecemos de información suficiente sobre los resultados de los diferentes hospitales o consultorios, porque nadie la ha tabulado o porque los intentos de transparentarla son vistos como una amenaza. Como no conocemos los resultados concretos de las acciones de diagnóstico y trata- miento, la práctica médica no puede ser evaluada ni controlada. Por otra parte, no existen mecanismos que obliguen a acreditar las destrezas de los médicos o las instituciones $\mathrm{y}$, a pesar de la influencia de la OPS en las reformas iniciadas en la década 1980-89 para favorecer al Estado como ente regulador, estamos lejos de una separación real y eficaz de las funciones del Ministerio de Salud.

El énfasis en el modelo biológico

Tanto la enseñanza como la práctica están centradas en los hospitales y en un modelo que privilegia los aspectos biológicos de la enfermedad (modelo bio-médico hospitalario). El enfermar es visto como un desorden orgánico y la especialización es fuertemente estimulada mientras los incentivos al médico general son mínimos, aunque sabemos que la especialización es la forma menos costo-eficaz de resolver los problemas de salud.

\section{La pérdida de sentido}

Un halo de sin-sentido envuelve mucho de lo que hacemos. La razón es que no hemos considerado suficientemente importantes los factores sociales, psicológicos, culturales y ambientales, en otras palabras: el contexto en el que ocurre la enfermedad. La experiencia de la crisis como un continuo de nuestra época, se ha trasladado a la experiencia de la enfermedad, que suele ser un modo de vivir creado y sostenido por la tecnología. Tenemos 10.000 pacientes en hemodiálisis crónica y su número crece $8 \%$ al año ${ }^{19}$. Las unidades de cuidados intensivos son percibidas como centros de alto nivel pero, también, como fuentes de grandes problemas éticos, sociales y económicos. La tecnología -necesaria en la cara exitosa del trabajo médico- resulta claramente insuficiente.

La incertidumbre y el temor Citamos de New England Journal of Medicine ${ }^{20}$ : «Alguna vez, la consulta fue la provincia privada del doctor. Ahora, mucho de lo que allí pasa es vigilado por terceros. La posibilidad de demandas causa una sensación de desagrado, las peticiones de hasta un tercio de los pacientes para llenar documentación escrita no vinculada al diagnóstico ni al tratamiento, es una causa de distracción y pérdida de tiempo. La pérdida de independencia y la reducción dela autonomía profesional, llevan a la insatisfacción y, si las restricciones son severas, a la desmoralización»? 
La otra cara de la moneda es un aumento insostenible de los costos, por lo cual, el autor propone un rediseño completo del sistema. En nuestro país es imperativo -para el proceso de reforma- profundizar el análisis, identificar correctamente los problemas y formular en forma precisa las preguntas antes de buscar las respuestas. Cuando las expectativas de salud de una comunidad son realizables, ella tiene derecho a influir en la forma en que se organiza la atención médica, pero tiene también el deber de hacer lo que le corresponde para cumplir con sus propios anhelos.

Hay otras causas de incertidumbre. Los cambios continuos en los métodos de diagnóstico y tratamiento hacen sentir a cada profesional que, cuanto más especializado es, más cercano está a su obsolescencia. Se habla de la robotización de ciertas prácticas. Aumenta el número de egresados y de médicos extranjeros y también la competencia entre los médicos y entre las instituciones. Lo más importante es el deterioro de la relación médico-paciente ${ }^{21}$. Parecemos un mal necesario. Mantenemos una actitud patemalista, no enfatizamos adecuadamente la importancia del auto-cuidado y las responsabilidades del paciente y su familia; tomamos todas las decisiones y, si algo sale mal, tendremos toda la «culpa».

Con razón el médico e historiador español Pedro Laín Entralgo ha descrito la medicina de nuestro tiempo con dos palabras: poderío y perplejidad $^{22}$. El inmenso poder del conocimiento actual y la perplejidad moral e intelectual al advertir que todo ese poder no será suficiente para resolver los problemas.

\section{LOS PROBLEMAS CON LOS FACTORES DETERMINANTES}

Las dificultades para absorber con eficiencia el rápido y continuo cambio científico-tecnológico fueron abordadas en el punto anterior. Ahora veremos las restantes.

1. Desde un punto de vista ético hay un debilitamiento; el paciente parece más un medio que un fin en sí mismo. Y los problemas éticos no pueden solucionarse trasladándolos al plano legal (o aumentando el uso del consentimiento informado). La mayoría de los médicos se ha quedado en la era hipocrática -en una ética de la prudencia y del principio de beneficencia- que parecía suficien- te, pero que nunca necesitó reflexionar sobre la relación médico-paciente. Los médicos hemos tenido poco que ofrecer en este campo. Fue a partir del pensamiento de los filósofos, quienes empezaron a reflexionar sobre los problemas de la ética médica desde la década 1960-69, que se desarrolló el concepto de bioética y la teoría principialista, que agregó los principios de justicia y autonomía al limitado marco con que contaba nuestra práctica ${ }^{23}$.

2. En el plano legal, no hemos sido capaces de seguir los cambios jurídicos para entender la actualidad del concepto de responsabilidad civil asociada a las complicaciones médicas y separarla de la idea de culpa. No consideramos de suficiente importancia aquello que no tiene relación directa con el diagnóstico y tratamiento y tenemos problemas para completar protocolos, registros y certificados. Existe una gran dispersión de criterios para otorgar licencias médicas, que no puede ser explicada por motivos técnicos.

3. En el ámbito económico, en todo el mundo occidental se repite un escenario que no calza con la ortodoxia: mayor gasto con menor satisfacción de los usuarios. Los profesionales del área económica ingresaron a la nuestra hace más de 20 años y sus demandas están adquiriendo una creciente autoridad, a la que los médicos se oponen sin suficientes herramientas. La mayor parte de las veces los conflictos son teóricos. En la vida real, cada médico se esfuerza por optimizar el uso de su tiempo con una lógica económica impecable. A su vez, los economistas que se enferman aspiran a ser tratados, como todo paciente, de forma especial siguiendo la lógica médica.

Sin embargo, dado el particular «campo» de trabajo del médico, que implica una relación con el enfermo y su entorno, más allá de un contrato, es un grave error reducir el trabajo médico a los términos de cualquier actividad productiva, en las cuales sí es posible un control central. Los intereses médicos están lejos de satisfacerse sólo con un pago. Los médicos atienden todos los días enfermos sin costo alguno, participan en organizaciones humanitarias, proyectos de investigación de nula rentabilidad y en la vida cívica, porque piensan que su quehacer encierra una enorme responsabilidad social y que su ámbito de influencia debería ir más allá de aquellas cosas para las cuales están verdaderamente entrenados. Pero, en realidad, tenemos innumerables problemas que 
comprender y resolver primero en nuestro propio campo.

4. En nuestras organizaciones hay confusión entre los asuntos gremiales, políticos y técnicos. El bienestar de los pacientes sirve para explicarlo todo, desde las demandas por mejor equipamiento hospitalario, hasta las huelgas motivadas económica 0 políticamente, lo que ha aumentado la suspicacia de los demás actores sociales. Y, a pesar de las dificultades que enfrentamos, participamos poco en las elecciones de nuestros representantes gremiales.

\section{Cómo LEgGamos A LA SITUACIÓN ACTUAL}

El sociólogo Gabriel Gyarmati, en un artículo publicado en la revista Vida Médica, recurriendo al análisis del historiador Arnold Toynbee señala:

«uando un grupo o sector social logra alto status o poder gracias a sus ideas creativas para enfrentar determinadas circunstancias históricas, tiende a aferrarse a los mismos métodos e ideas que lo llevaron al éxito aun cuando las circunstancias con el tiempo hayan cambiado. Esta rigidez, eventualmente, conduce a su desplazamiento y al surgimiento de nuevos grupos sociales, con ideas mejor adaptadas a los nuevos requerimientos, ${ }^{24}$.

Este parece ser nuestro caso. Toda innovación conceptual es percibida como una amenaza al status social y económico logrados con un modelo de trabajo que nos hizo exitosos. No hemos comprendido que, tanto la salud como la medicina, son hechos sociales y no estamos habituados a tratar científicamente con ellos. Los fenómenos sociales son modos de actuar, pensar o sentir externos al sujeto en que se presentan, pero que se le imponen, quiéralo o no, pues su fundamento no es el individuo ${ }^{25}$. La salud pertenece a esta esfera. Trasciende al quehacer y pensamiento médico y, en última instancia, se escapa de su control ya que la salud es, en primer lugar, una construcción personal y, al mismo tiempo, un bien social ${ }^{26}$.

Lo que nos hizo exitosos fue el desarmollo del modelo biomédico-hospitalario que, al incorporar los avances tecnológicos, pudo dar respuestas adecuadas a los problemas de salud. Este modelo generó nuevos problemas: desde la década 1950-59 se ha producido un aumento sostenido de las esperanzas de la población respecto de sus condiciones de salud. Intentar satisfacerlas significó insti- tucionalizar y socializar la atención médica ${ }^{27}$. La socialización de la atención, que requirió desarrollar los seguros de salud, logró la superación de muchos problemas pero las expectativas aumentaron. Y no dejarán de hacenlo, porque los avances tecnológicos continuarán y la salud se ha transformado en un derecho sin contrapeso en ningún deber.

En este contexto, el modelo señalado induce la especialización eficaz, que va acompañada de una fragmentación y despersonalización de la atención. El carácter explosivo de la situación se completa cuando la demanda no puede ser satisfecha debido a la restricción de los aportes fiscales, una consecuencia del crecimiento de los costos. Entonces, la población reacciona, pero especialmente contra los médicos. Lo anterior es crítico en el sector público porque éste ha perdido su capacidad de estimulary retener a los médicos. Pero el sector no estatal -producto de una reforma parcial al sistema de salud en la década 1980-89- no ha cambiado el modelo de atención y se puede prever, también en él, un aumento progresivo de los costos y el desarrollo de diversas formas de administrarlo para dar cuenta de las expectativas. No olvidemos que el éxito de las políticas de salud de la década 195059 fue producto de programas pensados, aplicados y finalmente controlados.

\section{CONDICIONES NECESARIAS PARA AVANZAR}

Debemos asumir el desarrollo tecnológico abordando, al mismo tiempo, los problemas «nternos» de la práctica profesional porque los cambios, aunque apremiados por presiones externas, sólo pueden venir de los propios médicos. Las instituciones que lideran el desarrollo médico deberían levantar una voz respetable y poderosa por su novedad y calidad.

Los elementos que enunciamos a continuación, parecen básicos: 1. La responsabilidad de mantener la salud debe centrarse no en los médicos, sino en la comunidad educada en aspectos claves de prevención primaria y secundaria y consciente de los límites del arte médico. Bajo ninguna circunstancia los médicos podremos mantener -en un ambiente lleno de excesos- la salud de las personas si éstas no consideran que la solución de los problemas prevalentes depende de ellas en primer lugar; 2. La costo-efectividad de 
las acciones de diagnóstico y tratamiento en nuestro medio, debe ser analizada para utilizar de mejor manera la infraestructura tecnológica; 3. El tiempo dedicado a la renovación conceptual -imprescindible para la actualización de la práctica- debería ser exigido, respetado y remunerado; 4. La remuneración de los médicos debe estar vinculada - de la manera que parezca más adecuada- con la cantidad y calidad de la actividad que realicen. Por lo tanto, cantidad y calidad deben ser

\section{REFERENCIAS}

1. Sмптн R. Why are doctors so unhappy? BMJ 2001; 322: 1073-74.

2. Chew M, Wiwams A. Australian General practitioner: desperately seeking satisfaction: is the satisfied GP an oxymoron? Med J Aust 2001; 175: 85-6.

3. KASSIRER JP. Doctor discontent. N Engl J M 1998; 339: 1543-5.

4. Edwards N, Kornacki MJ, Silversin J. Unhappy doctors: What are the causes and what can be done? BMJ 2002; 324: 835-6.

5. JimÉneZ de la JaRA J. Cambios en el entorno del trabajo médico. Rev Méd Chile 2004; 132: 637-42.

6. Horwitz N. El cambio en la práctica médica. Desafíos psicosociales para la profesión. Rev Méd Chile 2004; 132: 768-72.

7. Vukusich A. El Trabajo Médico en Chile. III Seminario anual de la Asociación de Clínicas. 2001; 13-21.

8. RoEsSiER E. Amenazas y oportunidades de la Sociedad Médica de Santiago en el Siglo XXI. Boletín de la Sociedad Médica de Santiago. 2004; Vol 7. № 1.

9. PiñeRA B. El reencantamiento de la Vida. Santiago: Editorial Los Andes, 1993; 17.

10. Descartes R. Discurso del método. Buenos Aires: Losada, 1966.

11. LAPLACE PS. Essai philosophique sur les probabilités. En Ouvres completes de Laplace. Paris: Academie des Sciences, 1812; 1878-1919. Vol. 7.

12. Hawking SW. Historia del Tiempo. Del Big Bang a los agujeros negros. Barcelona: Crítica, 1988.

13. Mosterín J, TorRetTI R. Diccionario de Lógica y Filosofía dela Ciencia. Madrid: Alianza, 2002; 549-51.

14. Heisenberg. Das Bild der Natur in der heutingen Physik. Traducción de G. Ferraté. En: Laín Entralgo, López Piñero. Panorama histórico de la Ciencia Moderna. Madrid: Guadarrama, 1973. medidas; 5. Debe documentarse con información científica la costo-efectividad de la atención primaria para hacer las correcciones a tiempo; 6 . Debemos conocer y comprender mejor cada uno de los factores que determinan nuestro quehacer, para actuar con inteligencia y eficacia ante los demás actores sociales. Buscar la solución en el pasado traerá de vuelta los mismos problemas que tenemos hoy. La situación es nueva. Debemos pensar de nuevo y actuar en consecuencia.

15 Hawking SW. El Universo en una Cáscara de Nuez. Barcelona: Crítica, 2002.

16. Nietzsche F. El Origen de la Tragedia. Madrid: Espasa Calpe, 1975.

17. VukUsich A, VaLdÉs S. La ficha clínica orientada por problemas. Rev Méd Chile 1988; 116: 1070-74.

18. Giuglano R, Lievadot J, Wilcox R, GuRfinkel E, Mc Cabe C, Charlesworth A et al. Geographic variation in patient and hospital characteristics, management and clinical outcomes in S-T elevation myocardial infarction treated with fibrinolysis: Results from in TIME-II. Euro Heart J 2001; 22: 1702-15.

19. Poblete H. Registro chileno de hemodiálisis. Sociedad Chilena de Nefrología. ASODI. 2004.

20. Campion E. A symptom of discontent. N Engl J Med 2001; 344: 223-25.

21. SeRANI A. La relación médico-paciente en un mundo de proveedores y clientes. En: Salud Privada: Problemas emergentes, alternativas de solución. Consejo Médico Asociación de Clínicas. 2000; $15-20$.

22. Laín P. Historia de la Medicina. Barcelona: Salvat, 1982; 461-560.

23. Peliegrino E. La metamorfosis de la ética médica. Una mirada retrospectiva a los últimos 30 años. Cuadernos Regionales del Programa de Bioética. OPS, OMS. 1995; 35-53.

24. Gyarmati G. La crisis de la medicina y el rol de la profesión médica en ella. Vida Médica. 1992; 44: 16-19.

25. DuRKheim E. Las reglas del método sociológico. Buenos Aires: La Pléyade 1979; 29-39.

26. Diario El Mercurio (ed). 1992. 28 de Noviembre.

27. JIMÉNEZ J. Historia de la organización de los servicios médicos y del financiamiento de la salud en Chile: Una visión personal. En: La Salud Privada en Chile: Desafíos para un Sistema. Consejo Médico de la Asociación de Clínicas. 1999; 4-16. 